\title{
A GENERALIZATION OF THE 5-COLOR THEOREM
}

\author{
PAUL C. KAINEN 1
}

ABSTRACT. We present a short topological proof of the 5-color theorem using only the nonplanarity of $K_{6}$. As a bonus, we find that any graph which becomes planar upon the removal of 2 edges can be 5-colored and that any graph which becomes planar when 5 edges are removed is 6 -colorable.

Let $G$ be a graph with no loops and no multiple edges joining the same pair of vertices. A 5-coloring of $G$ is a function $c$ which assigns to every vertex $v$ of $G$ a color $c(v)$ in the set $\{1,2,3,4,5\}$ and which satisfies the constraint $c(v) \neq c(w)$ whenever $v$ and $w$ are adjacent. A graph is planar if it can be embedded in the plane. We assume our graphs have a finite nonzero number of vertices. Our goal in this paper will be to present a short topological proof of Heawood's 5-color theorem [3] for planar graphs which leads to some interesting generalizations.

Theorem 1. Let $G$ be any planar graph. Then $G$ can be 5-colored.

Proof. Suppose the theorem is false. Then there is a planar graph $H$ with a minimal number $n$ of vertices which is not 5 -colorable. Since $H$ is planar, it contains a vertex $v$ of degree not exceeding 5. Let $H^{\prime}=H-v$. If the degree of $v$ is strictly less than 5 , then it is easy to see that any 5-coloring of $H^{\prime}$ extends to a 5-coloring of $H$. Moreover, by minimality of $H, H^{\prime}$ does have a 5-coloring. Hence, we may suppose that $v$ is adjacent to five vertices, $v_{1}, v_{2}, v_{3}, v_{4}, v_{5}$ enumerated in no special order. If every pair $v_{i}, v_{j}, 1 \leq i<j \leq 5$, were adjacent, then the set $\left\{v, v_{1}, \cdots, v_{5}\right\}$ would determine a complete subgraph $K_{6}$ of order 6 in $H$ which is impossible since $K_{6}$ is not planar. Hence, some pair of vertices, say $v_{1}, v_{2}$, are not adjacent. Let $\bar{H}$ be the graph obtained from $H^{\prime}$ by replacing $v_{1}$ and $v_{2}$ with a new vertex $\bar{v}$ which is adjacent to any vertex $w$ of $H^{\prime}$ to which

Received by the editors August 13, 1973.

AMS (MOS) subject classifications (1970). Primary 05C10, 05C15; Secondary 55A15.

Key words and phrases. Graph, chromatic number, 5-color theorem, skewness.

1 Research supported in part by NSF Grant GP 29551. 
either $v_{1}$ or $v_{2}$ was adjacent. The other vertices and adjacencies of $\bar{H}$ are the same as in $H^{\prime}$.

Let $e_{i}$ denote the edge $\left[v, v_{i}\right], 1 \leq i \leq 5$. Then $\bar{H}$ is planar since it can be obtained from $H$ by first deleting the edges $e_{3}, e_{4}$ and $e_{5}$, and then contracting the edges $e_{1}$ and $e_{2}$ removing any multiple edges which result. Since $\bar{H}$ has only $n-2$ vertices, it has a 5 -coloring $\bar{c}$. Defining $c^{\prime}\left(v_{1}\right)=\bar{c}(\bar{v})=c^{\prime}\left(v_{2}\right)$ and $c^{\prime}(w)=c(w)$ for any other vertex $w$ produces a 5-coloring $c^{\prime}$ of $H^{\prime}$ in which two of the vertices adjacent to $v$ receive the same color. Hence, $c^{\prime}$ extends to a 5-coloring $c$ of $H$. (Incidentally, we should mention that this approach is somewhat similar to arguments given by Birkhoff and by Grötzsch [2].)

Let us make three remarks about the proof of this theorem.

Remark 1. We needed to know that $K_{6}$ is not planar. (In fact, $K_{5}$ is not planar and so we have proved the 5 -color theorem without using the nonplanarity of $K_{5}$. One could regard this as a bit of propaganda for the 4-color conjecture.)

Remark 2. We also needed to know that any planar graph has a vertex of degree less than or equal to 5 . This is proved as follows. Suppose $G$ is planar with $n$ vertices and $m$ edges. Then $m \leq 3 n-6$ by Euler's formula. Let $d$ denote the average degree of $G$. Then, by definition, $d n=2 m$ so $d \leq 6-12 / n<6$. Hence, there is some vertex of degree $\leq 5$.

Remark 3. Finally, we needed to know that the class of planar graphs is closed under the operations of edge del etion and contraction.

We can make use of these three remarks to prove an interesting generalization of Theorem 1. Define the skewness $\mu(G)$ of a graph $G$ to be the minimum number of edges whose removal makes $G$ planar.

Theorem 2. Let $G$ be any graph with $\mu(G) \leq 2$. Then $G$ can be 5-colored.

Proof. The proof of Theorem 1 will go through provided that we can verify the analogues of Remarks 1,2, and 3 .

Lemma $1 . K_{6}$ does not have skewness $\leq 2$.

Proof. $K_{6}$ has 6 vertices and 15 edges so it must have skewness at least 3. Since it is easy to construct a planar embedding of $K_{6}$ minus 3 edges, $\mu\left(K_{6}\right)=3$.

Lemma 2. Suppose $\mu(G) \leq 2$. Then $G$ has a vertex of degree $\leq 5$.

Proof. Let $G$ have $n$ vertices and $m$ edges. Then $m-2 \leq 3 n-6$ so 
$m \leq 3 n-4$. Hence, as before, the average degree $d$ is less than 6 .

Unfortunately, it is not necessarily true that the class of graphs with skewness $\leq 2$ is closed under edge deletion and contraction. For if an edge $e$ which we want to contract must be del eted to make the graph planar, then, once $e$ is contracted, it becomes a vertex whose removal is no longer allowed. Nevertheless, we can still push through the proof.

As before, let $H$ be a minimal non-5-colorable graph satisfying $\mu(H) \leq$ 2. Suppose $H$ has $n$ vertices and $m$ edges. If $n_{i}$ is the number of vertices of degree $i$, then by minimality $n_{i}=0$ for $i<5, n=\Sigma_{i} n_{i}$, and $2 m=\Sigma_{i} i n_{i}$. Moreover, by Lemma $2, m \leq 3 n-4$ so

$$
6 n-8=-8+\sum_{i} 6 n_{i} \geq \sum_{i} i n_{i}
$$

or

$$
8 \leq \sum_{i}(6-i) n_{i}=n_{5}-n_{7}-2 n_{8}-\cdots
$$

Hence, we have proven the analogue of a famous lemma of Franklin [1].

Lemma 3. Let $H$ be a minimal non-5-colorable graph satisfying $\mu(H)$ $\leq 2$. Then $H$ has at least 8 vertices of degree 5 .

We can use this lemma to complete the proof of Theorem 2. Choose two edges $e$ and $e^{\prime}$ in $H$ whose deletion makes $H$ planar. These edges are incident with at most 4 vertices and so we can choose a vertex $v$ in $H$ of degree 5 and not incident with either $e$ or $e^{\prime}$. Now deleting and contracting edges incident with $v$ produces a new graph $\bar{H}$ whose skewness does not exceed 2 since $\bar{H}-\left\{e, e^{\prime}\right\}$ is the planar graph obtained from $H-\left\{e, e^{\prime}\right\}$ by deletion and contraction of edges. This completes the proof of Theorem 2.

By the proof of Lemma $1, \mu\left(K_{6}\right)=3$, and certainly $K_{6}$ cannot be 5-colored. Thus, Theorem 2 is best possible. The following result is proved just like Lemma 2, of which it is a strengthening.

Lemma 4. If $\mu(G) \leq 5$, then $G$ has a vertex of degree $\leq 5$.

Using this lemma, we can generalize Theorem 1 further provided we weaken 5-colorable to 6-colorable.

Theorem 3. Every graph $G$ with $\mu(G) \leq 5$ can be 6-colored.

Proof. Let $H$ be a minimal non-6-colorable graph with $\mu(H) \leq 5$. By Lemma $4, H$ has a vertex $v$ with degree $\leq 5$. Since $\mu(H-v) \leq \mu(H) \leq 5$, $H-v$ has a 6-coloring which necessarily extends to $H$. 
This result is also best possible since $\mu\left(K_{7}\right)=6$.

\section{REFERENCES}

1. P. Franklin, The four color problem, Scripta Math. 6 (1939), 149-156, 197210. MR 1, 316.

2. H. Grötzsch, Zur Theorie der diskreten Gebilde. VII. Ein Dreifarbensatz für dreikreisfreie Netze auf der Kugel, Wiss. Z. Martin-Luther-Univ. Halle-Wittenberg. Math.-Nat. Reihe 8 (1958/59), 109-120. MR 22 \#7113c.

3. P. J. Heawood, Map colour theorem, Quart. J. Math. 24 (1890), 332-338.

DEPARTMENT OF MATHEMATICS, CASE WESTERN RESERVE UNIVERSITY, CLEVELAND, OHIO 44106 